\title{
Early-stage tumor detection using photoacoustic microscopy: a pattern recognition approach
}

Chenghung Yeh, Liang Wang, Jinyang Liang, Yong Zhou, Song $\mathrm{Hu}$, et al.

Chenghung Yeh, Liang Wang, Jinyang Liang, Yong Zhou, Song Hu, Rebecca E. Sohn, Jeffrey M. Arbeit, Lihong V. Wang, "Early-stage tumor detection using photoacoustic microscopy: a pattern recognition approach," Proc. SPIE 10064, Photons Plus Ultrasound: Imaging and Sensing 2017, 100644N (3 March 2017); doi: 10.1117/12.2253529

\section{SPIE. Event: SPIE BiOS, 2017, San Francisco, California, United States}




\title{
Early-stage tumor detection using photoacoustic microscopy:
}

\section{A pattern recognition approach}

\author{
Chenghung Yeh ${ }^{1}$, Liang Wang ${ }^{1}$, Jinyang Liang ${ }^{2}$, Yong Zhou ${ }^{2}$, Song $\mathrm{Hu}^{3}$, \\ Rebecca E. Sohn', Jeffrey M. Arbeit ${ }^{4, *}$ and Lihong V. Wang ${ }^{1,2, *}$ \\ ${ }^{1}$ Department of Electrical and Systems Engineering, Washington University in St. \\ Louis, One Brookings Dr., St. Louis, MO, 63130 \\ ${ }^{2}$ Department of Biomedical Engineering, Washington University in St. Louis, One \\ Brookings Dr., St. Louis, MO, 63130 \\ ${ }^{3}$ Department of Biomedical Engineering, University of Virginia, \\ PO Box 800759, Charlottesville, VA 22908, USA \\ ${ }^{4}$ Urology Division, Department of Surgery, Washington University School of \\ Medicine, St. Louis, MO 63130, USA \\ *Corresponding authors: arbeitj@wust.edu (animal model); lihong@caltech.edu
}

(microscopy)

\begin{abstract}
We report photoacoustic microscopy (PAM) of arteriovenous (AV) shunts in early stage tumors in vivo, and develop a pattern recognition framework for computerized tumor detection. Here, using a high-resolution photoacoustic microscope, we implement a new blood oxygenation $\left(\mathrm{sO}_{2}\right)$-based disease marker induced by the AV shunt effect in tumor angiogenesis. We discovered a striking biological phenomenon: There can be two dramatically different $\mathrm{sO}_{2}$ values in bloodstreams flowing side-by-side in a single vessel. By tracing abnormal $\mathrm{sO}_{2}$ values in the blood vessels, we can identify a tumor region at an early stage. To further automate tumor detection based on our findings, we adopt widely used pattern recognition methods and develop an efficient computerized classification framework. The test result shows over $80 \%$ averaged detection accuracy with false positive contributing $18.52 \%$ of error test samples on a 50 PAM image dataset.
\end{abstract}

Keywords: photoacoustic microscopy, optical-resolution, oxygen saturation, tumor, cancer, arteriovenous shunt, pattern recognition, machine learning

Photons Plus Ultrasound: Imaging and Sensing 2017, edited by Alexander A. Oraevsky, Lihong V. Wang, Proc. of SPIE Vol. 10064, 100644N · C 2017 SPIE · CCC code: 1605-7422/17/\$18 · doi: 10.1117/12.2253529 


\section{INTRODUCTION}

As a cancerous tumor starts to grow in tissue, the tumor cells stimulate a vessel network to support their growth - this process is called angiogenesis. In the cardiovascular system, arteries transport oxygenated hemoglobin and then deoxygenated hemoglobin is carried away by veins. Vessel remodeling caused by angiogenesis leads to the development of arteriovenous (AV) shunts - a short pathway between arteries and veins. This process leads to abnormally high oxygenated hemoglobin in veins and may alter drug delivery efficiency [1]. Recently, treating tumor growth by normalizing the tumor vessel network has attracted much interests [2,3]. However, early detection of the AV shunt effect in tumor has not been well explored yet.

Photoacoustic tomography (PAT), combining with optical excitation and ultrasonic detection, is a powerful tool for providing multiscale images of living biological structures [4-8]. Optical resolution photoacoustic microscopy (OR-PAM), which is one version of PAT, uses endogenous hemoglobin absorption contrast to image the microvasculature [9-11]. The detailed images provided by OR-PAM pose interesting challenges in interpretation and classification. In recent years, automated decision making using machine learning and statistical methods has seen an explosion of interest in modern medical computing settings, especially in different imaging modalities, such as EEG [12], MRI [13]. Advanced algorithms are being developed to harvest insights from large image datasets. In this paper, based on well-developed techniques in computer vision and machine learning, we developed an automatic tumor pattern classification framework for OR-PAM images and we believe our framework would encourage more research in algorithms and software for PAT.

\section{METHODS AND MATERIALS}

\subsection{OR-PAM}

Our OR-PAM system [14, 15] (Fig. 1) uses a nanosecond-pulsed solid-state laser (INNOSLAB, Edgewave, 532 $\mathrm{nm}$ and $559 \mathrm{~nm}$ wavelengths; $30 \mathrm{kHz}$ pulse repetition rate) [10]. First, an iris spatially filters the laser beam, which then passes through a spatial filtering stage consisting of a condenser lens (LA1131, Thorlabs) and a pinhole (P50C, Thorlabs). The spatially filtered beam is then coupled into a single-mode photonic crystal fiber (LMA-10, NKT Photonics) by an objective lens $(4 \times, \mathrm{NA}=0.1$, Leica). The output laser beam is collimated by an objective lens (RMS4×, Thorlabs) and then focused by another identical objective lens onto the imaging target. The generated photoacoustic waves are detected by an ultrasonic transducer (V214-BB-RM, Olympus-NDT).

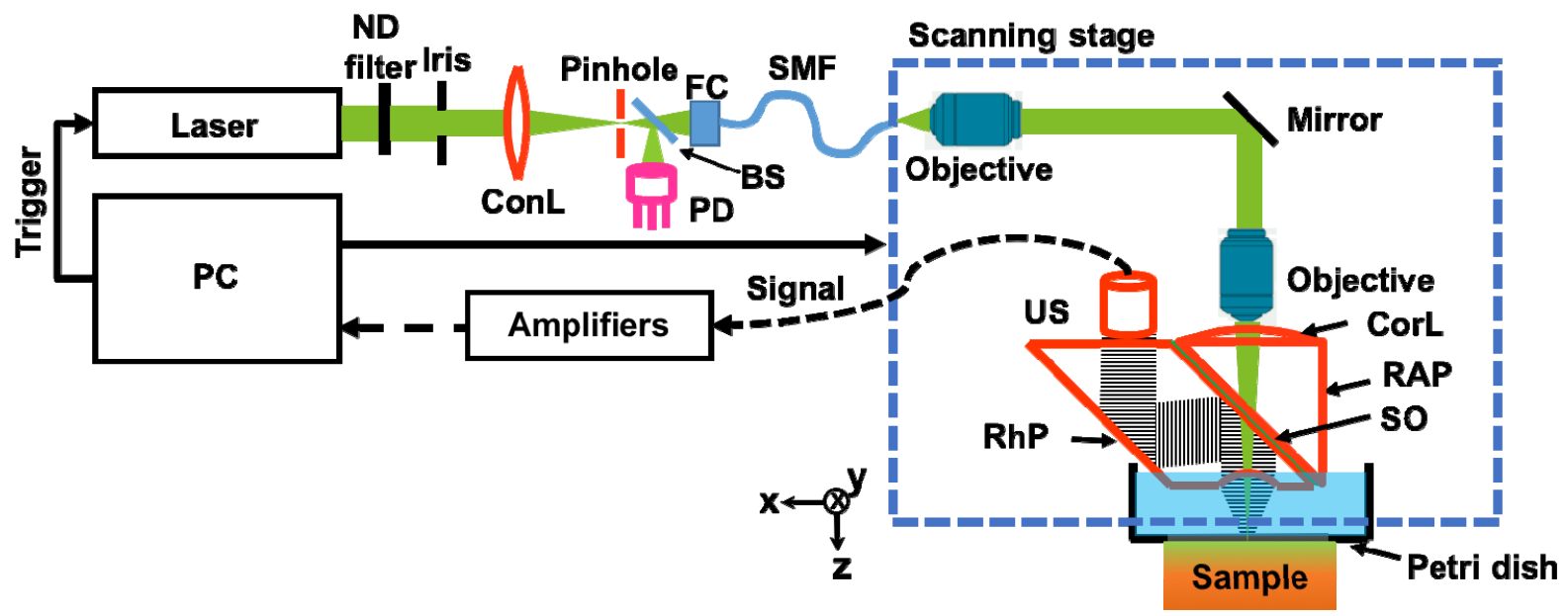

Figure 1. Schematic diagram of OR-PAM. BS, beam splitter; ConL, condenser lens; CorL, correction lens; FC, fiber coupler filter; ND, neutral density; PD, photodiode; RAP, right-angle prism; RhP, rhomboid prism; SMF, single-mode fiber; SO, silicone oil; US, ultrasonic transducer. 
a

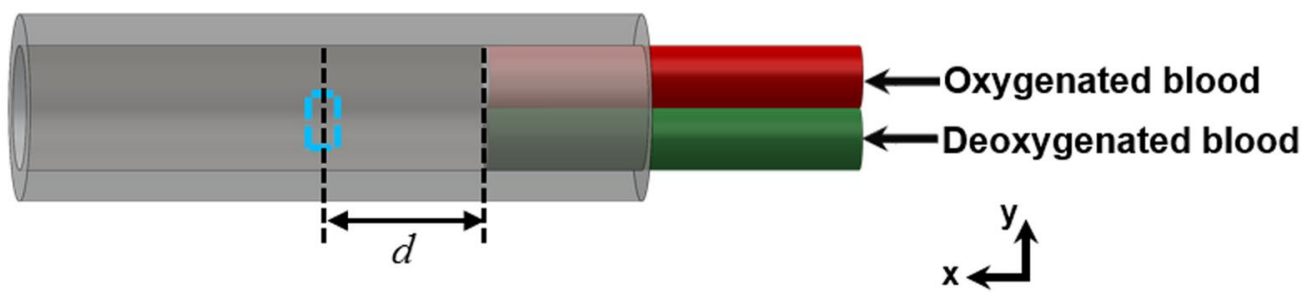

b

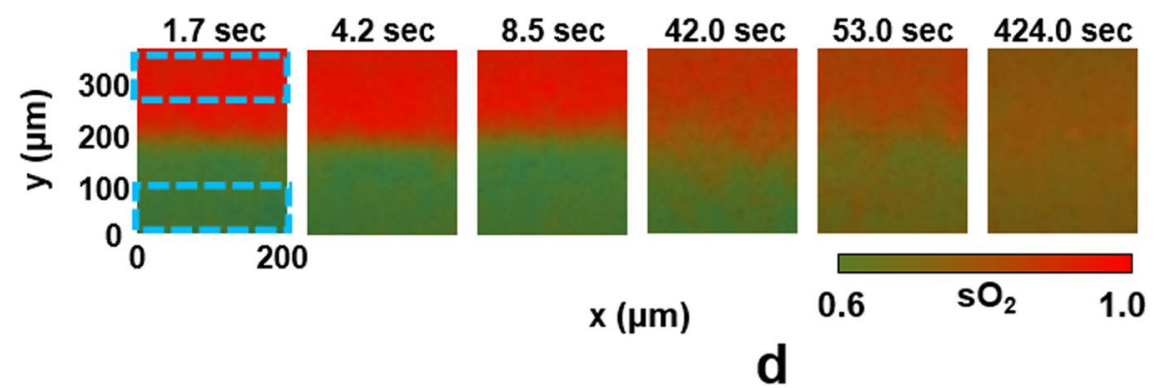

C

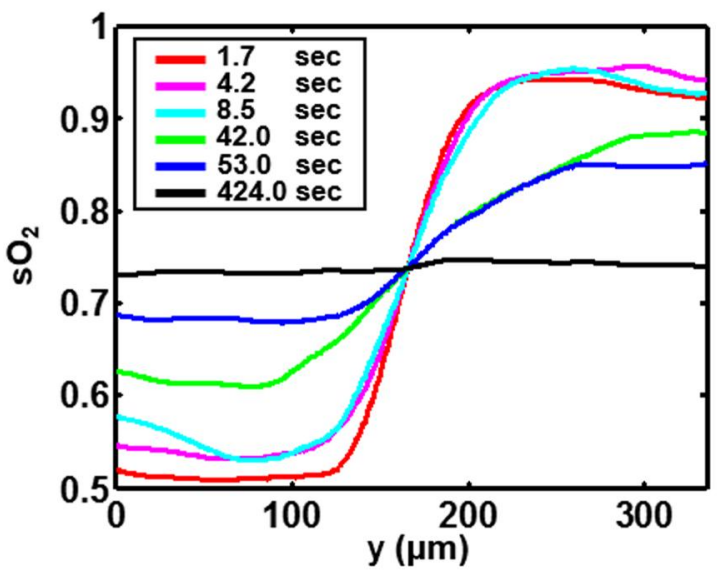

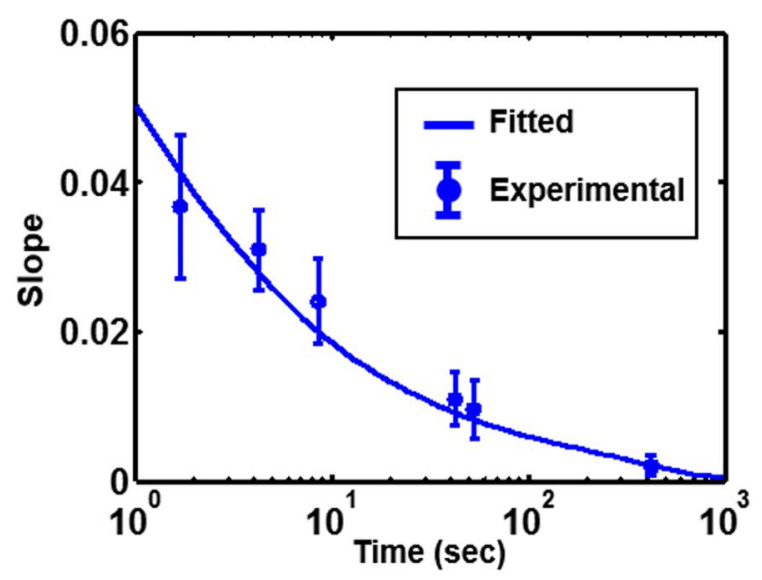

Fig. 2. OR-PAM of the AV shunt effect in a phantom. (a) Schematic of phantom. Two smaller-diameter tubes (ID $187.5 \mu \mathrm{m}$, OD $250 \mu \mathrm{m}$ ) are inserted into one larger-diameter tube (ID $500 \mu \mathrm{m}$, OD $750 \mu \mathrm{m}$ ). One small tube was injected with oxygenated blood and the other with deoxygenated blood. The co-current blood flow was controlled by a syringe pump for each small tube. The dashed blue boxes indicates the imaging regions of interest. (b) Measured $\mathrm{sO}_{2}$ distributions at 1.7, 4.2, 8.5, 42.0, 53.0, and 424.0 seconds. (c) $\mathrm{sO}_{2}$ averaged along $x$ versus $y$ at 1.7, 4.2, 8.5, 42.0, 53.0, and 424.0 seconds. (d) Measured $\mathrm{sO}_{2}$ slopes along the $y$ direction at the center of the tube versus time and fit based on a diffusion model. 


\section{a}

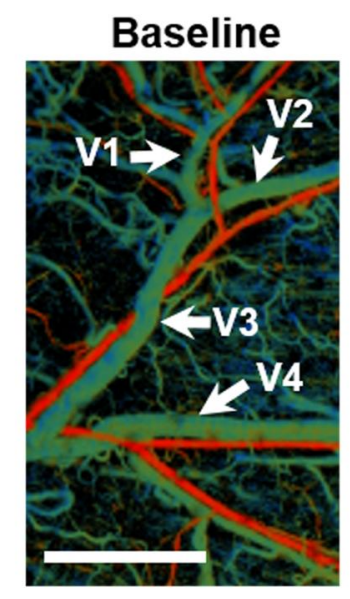

b

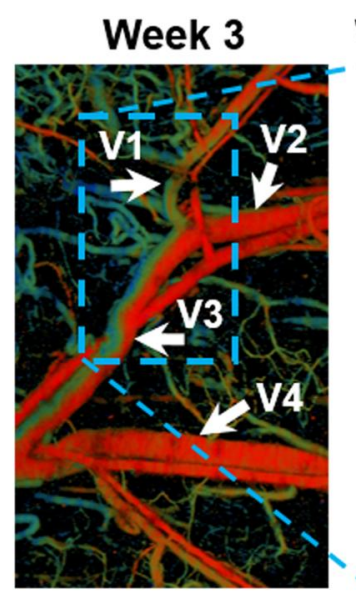

Week 3 highlight

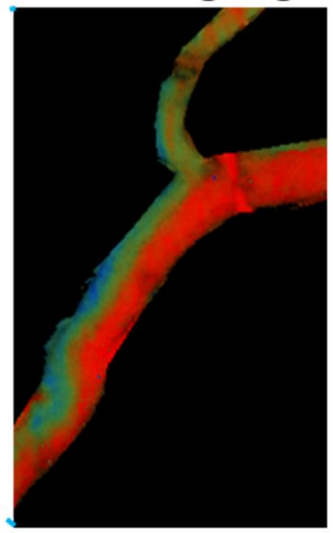

C

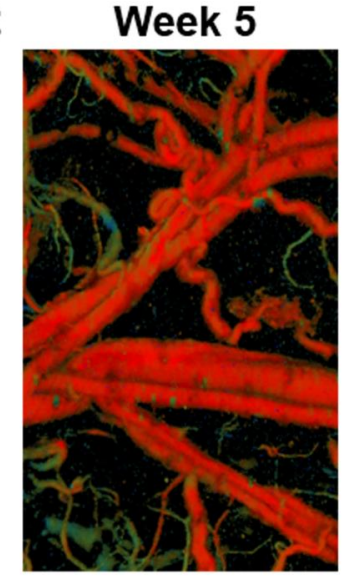

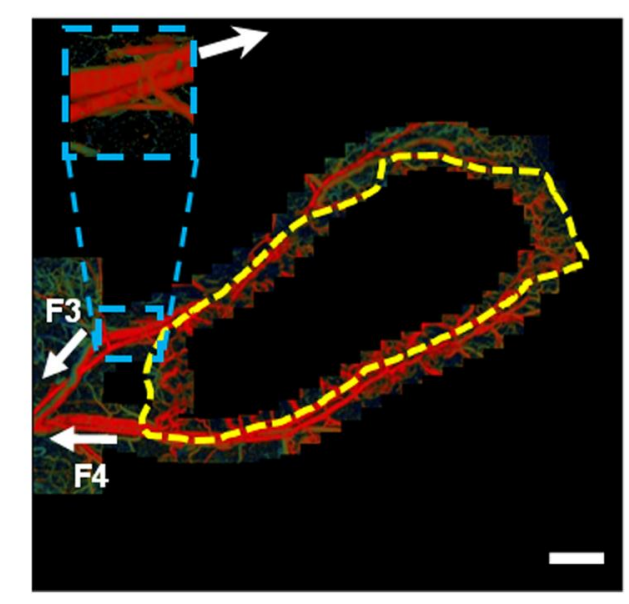

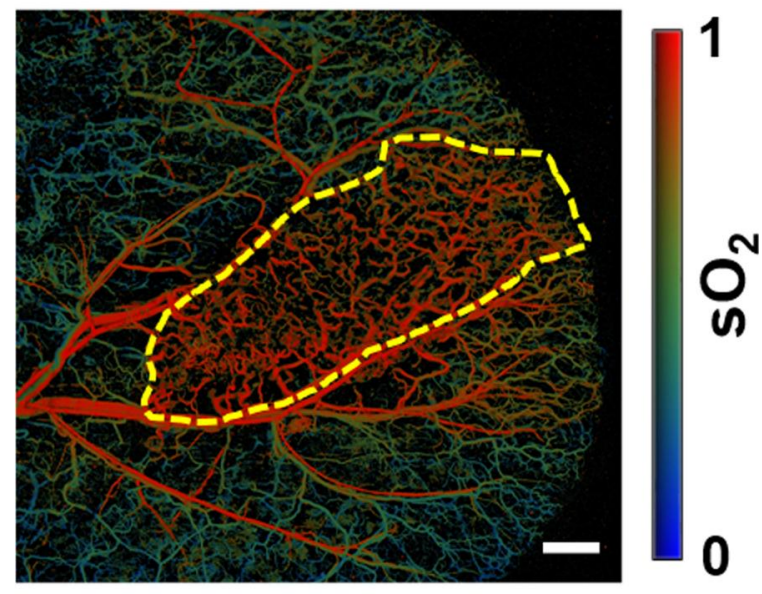

Fig. 3. OR-PAM of the AV shunt effect in a mouse ear in vivo. (a) $\mathrm{sO}_{2}$ images of a mouse ear acquired before (baseline), 3 weeks after, and 5 weeks after injection of xenotransplanted O-786 cells. The blue dashed box encloses a highlighted region. V1, normal vessel; V2, week-3 abnormal vessel; V3, confluence vessel of V1 and V2; V4, week-3 high $\mathrm{sO}_{2}$ vein. (b) Tiled image of the $\mathrm{sO}_{2}$ map in a mouse ear using a three-dimensional arbitrary trajectory scan (the corresponding movie is Supplementary Video 1). The yellow dashed line bounds the predicted tumor region. $\mathrm{F} 3$ and $\mathrm{F} 4$, flow directions of veins $\mathrm{V} 3$ and $\mathrm{V} 4$. (c) Whole-ear $\mathrm{sO}_{2}$ map imaged by raster scan. The yellow dashed line region encloses the tumor region. Scale bars, $1 \mathrm{~mm}$.

\subsection{Phantom experiment design}

To mimic AV shunts and validate the physical mechanism of the confluence of an abnormal vein and a normal vein, we fabricated a polycarbonate bifurcation tube phantom (Fig. 2a), which connected two identical tubes (ID $187.5 \mu \mathrm{m}$, OD $250 \mu \mathrm{m}$; CTPC187-250, Paradigm Optics) and a larger-diameter tube (ID $500 \mu \mathrm{m}$, OD $750 \mu \mathrm{m}$; CTPC500-750, Paradigm Optics). We used the homemade tube structure to model the upstream veins and the downstream vein, respectively. The junction of the three tubes was sealed by glue. The other ends of the two smaller-diameter tubers were sealed to two individual syringes, each pushed by a syringe pump (NE-300, Pump Systems, Inc.). High $\mathrm{sO}_{2}(0.90)$ and low $\mathrm{sO}_{2}(0.54)$ blood flows were used in the two smaller-diameter tubes, and then the blood was pumped into the confluence tube. 
Our goal was to acquire the time course of the $\mathrm{sO}_{2}$ distribution at given the blood flow speed. One approach was to scan a large region of the confluence downstream vessel region. However, the OR-PAM system imposed a limited field of view $\left(20 \times 20 \mathrm{~mm}^{2}\right)$, which corresponded to a limited time window. Instead, we scanned an area of interest on the tube $\left(2.5 \mu \mathrm{m} \times 5.0 \mu \mathrm{m}\right.$ step size; $200 \times 365 \mu \mathrm{m}^{2}$ image size, marked by the dashed box in Fig. $2 a)$, located at a distance $(3 \mathrm{~mm})$ from the confluence point. Then we varied the upstream blood flow speed $(v)$ from $7.1 \times 10^{-3} \mathrm{~mm} / \mathrm{s}$ to $1.8 \mathrm{~mm} / \mathrm{s}$, which gave the $\mathrm{sO}_{2}$ distribution at various effective time points $(t=d / v)$, as shown in Fig. $2 \mathrm{~b}(1.7,4.2,8.5,42.0,53.0$, and 424.0 seconds).

\section{$2.3 \mathrm{sO}_{2}$ distribution model}

We built an analytical model based on Fick's law of diffusion $[16,17]$ to study the $\mathrm{sO}_{2}$ distribution along the cross section of the confluence vessel (the $y$ axis in Fig. 2a):

$$
J=-D_{\text {eff }} \frac{\partial\left[\mathrm{O}_{2}\right]_{\text {bound }}}{\partial y}, \text { (Eq. 1) }
$$

where $J, D_{\text {eff }}$, and $\left[\mathrm{O}_{2}\right]_{\text {bound }}$ denote the total flux of oxygen along the $y$ axis, the effective oxygen diffusion coefficient [18], and bound oxygen concentration, respectively. The effective oxygen diffusion coefficient is approximately modeled by

$$
D_{\text {eff }} \approx D_{R B C}+\frac{\alpha \times \beta^{\prime}}{\left[\mathrm{O}_{2}\right]_{\text {bound-max }}} \times D_{\text {oxygen }} \text {, (Eq. 2) }
$$

where $\alpha, \beta$, and $\left[\mathrm{O}_{2}\right]_{\text {bound-max }}$ respectively denote the solubility of oxygen, the slope of the quasi-linear portion of the oxygen dissociation curve (i.e., the portion between $P_{43}$ and $P_{91}$ ), and the maximum concentration of oxygen carried by RBCs. We used the thin-film approximation[19] for the $\mathrm{sO}_{2}$ distribution $c(y, t)$ along the cross section of the confluence vessel:

$$
c(y, t)=\frac{1}{\sqrt{4 \pi D_{e f f} t}} \int_{0}^{l} c\left(y^{\prime}, 0\right) \exp \left[\frac{-\left(y-y^{\prime}\right)^{2}}{4 D_{e f f} t}\right] \mathrm{d} y^{\prime}
$$

where $l$ denotes the diameter of the tube.

\subsection{Parameter}

We averaged the measured $\mathrm{sO}_{2}$ values at each time point (Fig. 2b) along the $x$ axis (Fig. 2c). The flux of oxygen is proportional according to $\mathrm{sO}_{2}$ slope (Eq. 1), so the maximum slope of each $\mathrm{sO}_{2}$ curve with respect to $y$ was chosen to quantify the diffusion speed. We fitted the PAM-measured $\mathrm{sO}_{2}$ slopes with an effective diffusion coefficient $\left(D_{\text {eff }}=7.5 \times 10^{-7} \mathrm{~cm}^{2} / \mathrm{s}\right)$ according to Eq. 3, as shown in Fig. $2 \mathrm{~d}$. Interestingly, the fitted effective diffusion coefficient is larger than the RBC diffusion coefficient $\left(D_{R B C}=4 \times 10^{-7} \mathrm{~cm}^{2} / \mathrm{s}\right)$ but smaller than the free oxygen diffusion coefficient $\left(D_{\text {oxygen }}=1.5 \times 10^{-5} \mathrm{~cm}^{2} / \mathrm{s}\right)$ reported in the literature $[18,20]$. This result is likely explained by the fact that most oxygen $(98.5 \%)$ is carried by RBCs, and only a small amount of free oxygen $(1.5 \%)$ is dissolved in the blood. We calculated $D_{\text {eff }} \approx 8.0 \times 10^{-7} \mathrm{~cm}^{2} / \mathrm{s}$ from Eq. 2 , and this value was comparable to the fitted value. We used this model and these parameters to predict that a distinguishable $\mathrm{sO}_{2}$ boundary can exist for $\sim 100$ seconds in a $100-\mu \mathrm{m}$-diameter vessel when the initial $\mathrm{sO}_{2}$ concentration difference is 0.36 . Note that the $\mathrm{sO}_{2}$ boundary is considered distinguishable if the difference in $\mathrm{sO}_{2}$ between these two areas is three times greater than the noise level. The noise level was defined by averaging the standard deviations over the undiffused high $\mathrm{sO}_{2}$ and low $\mathrm{sO}_{2}$ areas (dashed boxes in Fig. 2b). As the normal venous blood flow speed in mice is $5 \mathrm{~mm} / \mathrm{s}$ [21], this $\mathrm{sO}_{2}$ boundary should be able to last for as long as $500 \mathrm{~mm}$.

\subsection{Ethical review of procedures}

All experimental animal procedures were carried out in conformance with the laboratory animal protocol approved by the School of Medicine Animal Studies Committee of Washington University in St. Louis. 


\subsection{Tumor xenograft model}

786-O renal cell carcinoma cells $\left(1 \times 10^{6}\right.$ cells in $10 \mu \mathrm{L}$ of RPMI basal medium, CRL-1932, ATCC, Manassas, VA) were injected subcutaneously into one ear of a 6-8 week old nude mouse (Hsd:Athymic Nude-Foxn 1NU, Harlan Co.).

\subsection{Pattern recognition framework}

Our pattern recognition framework consists of four steps: In step 1, we decompose our PAT images containing ROI into wavelet basis functions. The motivation is to provide an effective method for tumor region edge detection. In step 2, we apply singular value decomposition (SVD) to find the principal components of these wavelet expanded images. This step can also be understood as a dimensionality reduction of our image feature vectors. In step 3, a linear statistical decision threshold is found using linear discrimination analysis (LDA) by projecting our features into appropriate subspaces. Finally, in step 4, the algorithms efficiency and accuracy are tested by running it 100 times on randomly generated cross-validation training and test datasets.
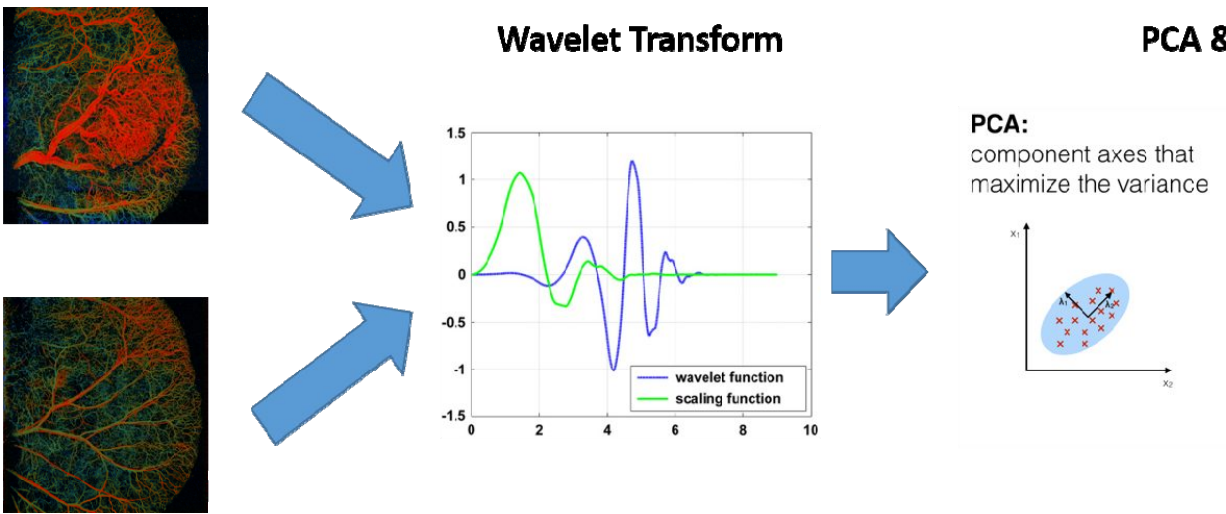

PCA \& LDA

Fig. 4 Flowchart of our pattern recognition framework.

PCA \& LDA image source: http://sebastianraschka.com/images/blog/2014/linear-discriminant-analysis/lda_1.png

\section{RESULTS AND DISCUSSION}

\subsection{In vivo demonstration}

To demonstrate that the $\mathrm{sO}_{2}$ boundary can be useful for tumor studies, we imaged O-786 xenograft tumor in one ear of four mice. OR-PAM monitored the $\mathrm{sO}_{2}$ distribution once per week for six weeks. All of four vasculatures had similar $\mathrm{sO}_{2}$ landscapes during their development. Fig. 3 shows the $\mathrm{sO}_{2}$ map of a mouse ear at three different time points: the baseline before, 3 weeks after, and 5 weeks after the tumor xenograft. First, we imaged the first bifurcation of the main vessel pair. The baseline image shows that $\mathrm{sO}_{2}$ values at the trunk vessel region in a healthy mouse ear are $\sim 0.90$ for arteries and $\sim 0.55$ for veins (Fig. 3a, left), which agrees well with the literature [21]. The week 3 image shows abnormal $\mathrm{sO}_{2}$ distribution, and higher $\mathrm{sO}_{2}$ values in portions of the veins (Fig. 3a, middle). Finally, the week 5 image shows a fully established tumor and abnormal $\mathrm{sO}_{2}$ affecting the whole imaged region (Fig. 3a, right). Moreover, the flow speed and diameter of the confluence vein (V3) were quantified to be $5 \mathrm{~mm} / \mathrm{s}$ and $180 \mu \mathrm{m}$, respectively. Using Eq. 2, it should take 185 seconds for the $\mathrm{sO}_{2}$ boundary to disappear in the confluence vessel, corresponding to an $\sim 900 \mathrm{~mm}$ distance at the given blood flow speed (5 $\mathrm{mm} / \mathrm{s}$ ). Moreover, we took a closer look at the confluence vein (V3), and we found a clear $\mathrm{sO}_{2}$ boundary, which was maintained in the imaged vessel $(\sim 1.5 \mathrm{~mm})$.

\subsection{Tumor detection}

These results pointed out a new way to detect abnormal blood vessels at a spot away from the tumor region and to trace these vessels back to the tumor region. To validate our approach, we raster scanned a region $(\sim 1.25 \mathrm{~mm}$ $\times 4.00 \mathrm{~mm}$ ) of the trunk vessel. We labeled the abnormal trunk vessels (V3 and V4) at week 3 in Fig. 3a, and 
identified the blood flows' directions (F3 and F4) in Fig 3b. Then, we performed a three-dimensional arbitrary trajectory scan [10], which allowed 3D position adjustment to trace the targeted vessels. By tracing these abnormal vessels against the flow direction to the upstream vessels, we identified the healthy region and abnormal region based on $\mathrm{sO}_{2}$ values (threshold $\mathrm{sO}_{2}$ : the mean of the values for a normal artery and vein). Next, we moved the scanning region by a small step $(0.35 \mathrm{~mm})$ tangential to the targeted vessel, and repeated the procedures above. Finally, all images were put together to form Fig. 3b. We predicted the tumor region (yellow dashed circle) by choosing the region with an abnormal high $\mathrm{SO}_{2}$ value. To verify our prediction, we scanned a whole ear (Fig. 3c) to identify the tumor region (yellow dashed circle in Fig. 3c), which is identified by vessels with abnormally high $\mathrm{sO}_{2}$ values and high vessel density [21].

\subsection{Pattern recognition of $i n$ vivo images}

To enhance the ability of the tumor detection, we implemented a pattern recognition based on our photoacoustic image [22]. Motivated by our preliminary observations of the tumor region and abnormal blood vessels, we collected a dataset of 50 images covering all tumor development stages in the mouse ear. By labeling the images with tumor as "1" and images without tumor as " 0 ", we essentially formulated our tumor pattern recognition problem as a binary classification problem. Thanks to rapid developments in machine learning and pattern recognition, mathematically plausible methods are being developed to mimic real biological processes. The goal is to use computers to automatically recognize animals, scenes, faces, or other complicated targets in images. Here, we adopt one simple and efficient approach that combines wavelet decomposition, PCA (principle component analysis), and LDA (linear discriminant analysis) for automatic tumor detection in blood vessel ORPAM images.

After cleaning and basic preprocessing of the image dataset, we got 41 images with the class label "1" and 9 images with the class label "0". We defined our ROI (region of interest) based on the location of blood vessels, and for simplicity we used a rectangular ROI with dimensions $650 \times 1100$ pixels. The detailed procedure of our algorithm is as follows:

(1) Wavelet decomposition

Wavelet analysis is known to be an ideal way to represent multi-scale information. Accordingly, wavelets were used here to detect and highlight edges of the tumor region. As seen in Fig. 3(c), finding the edge of tumor region could be a critical aspect of the discrimination process. In our implementation, we used the Haar wavelet as the wavelet basis function.

(2) PCA

PCA is a statistical procedure that uses orthogonal transformation to convert a set of observations of possibly correlated variables into a set of values of linearly uncorrelated variables. The principal components are ordered based on variance from largest to smallest. We implemented SVD (singular value decomposition) and chose the top 20 principal components as our feature for each sample.

(3) LDA

The final step in the training algorithm is to utilize this statistical information from wavelet/PCA in order to make a decision about whether a given image shows a tumor or not. LDA is a machine learning algorithm that finds a suitable projection that maximizes the distance between the inter-class data while minimizing the intra-class data.

Due to the limited of total number of images and the imbalance of tumor and no-tumor samples, we ran our algorithms 100 times on a random training set. During each run, the training set contained 8 random no-tumor samples and 30 random tumor samples, while the test set included all the remaining tumor and no-tumor samples. The averaged accuracy of 100 runs was $82.67 \%$. Also among the 100 runs, there are a total of 40 false positive test samples and a total of 176 false negative test samples. So false positive contributes to $18.52 \%$ of total error cases. This could be partly due to the imbalance of our tumor and no-tumor samples. The total running time was $\sim 260$ second in Matlab R2015b on a desktop computer with an Intel i7-5820k CPU and 32GB memory. This fast computation demonstrates the applicability of our automatic tumor detection algorithm based on photoacoustic images. 


\section{CONCLUSIONS}

OR-PAM can noninvasively measure $\mathrm{sO}_{2}$ with high spatial resolution and can detect the $\mathrm{AV}$ shunt effect in an early tumor region. Our theoretical model and phantom experiments indicated that the $\mathrm{sO}_{2}$ boundary induced by the AV shunt can exist for around $900 \mathrm{~mm}$ at the vessel parameters in the in vivo experiments. We successfully traced an early tumor in a mouse ear in vivo by using the $\mathrm{sO}_{2}$ distribution. To achieve automatic detection of tumors based on our finding, we developed a novel framework based on pattern recognition algorithms, and achieved a tumor identification accuracy of $82.67 \%$. We expect that this new discovery will find more applications, such as tracing $\mathrm{sO}_{2}$-based biomarkers in internal organs [7] and the brain in humans [23].

\section{ACKNOWLEDGEMENTS}

The authors appreciate the close reading of the manuscript by Prof. James Ballard. We also thank Cheng Ma, Pengfei Hai, and Hsun-Chia Hsu for helpful discussions. This work was sponsored by National Institutes of Health Grants DP1 EB016986 (NIH Director's Pioneer Award), R01 CA186567 (NIH Director's Transformative Research Award), and R01 CA159959.

\section{REFERENCES}

[1] A. R. Pries, M. Höpfner, F. le Noble et al., "The shunt problem: control of functional shunting in normal and tumour vasculature," Nat Rev Cancer, 10(8), 587-593 (2010).

[2] R. K. Jain, "Normalization of Tumor Vasculature: An Emerging Concept in Antiangiogenic Therapy," Science, 307(5706), 58-62 (2005).

[3] R. K. Jain, "Lessons from multidisciplinary translational trials on anti-angiogenic therapy of cancer," Nat Rev Cancer, 8(4), 309-316 (2008).

[4] L. V. Wang, and S. Hu, "Photoacoustic Tomography: In Vivo Imaging from Organelles to Organs," Science, 335(6075), 1458-1462 (2012).

[5] S.-L. Chen, Z. Xie, P. L. Carson et al., "In vivo flow speed measurement of capillaries by photoacoustic correlation spectroscopy," Optics Letters, 36(20), 4017-4019 (2011).

[6] Y. Zhou, X. Yi, W. Xing et al., "Microcirculatory changes identified by photoacoustic microscopy in patients with complex regional pain syndrome type I after stellate ganglion blocks," Journal of biomedical optics, 19(8), 086017-086017 (2014).

[7] C. Li, J.-M. Yang, R. Chen et al., "Urogenital photoacoustic endoscope," Optics Letters, 39(6), 1473-1476 (2014).

[8] R. J. Zemp, "Quantitative photoacoustic tomography with multiple optical sources," Applied optics, 49(18), 3566-3572 (2010).

[9] C. Yeh, S. Hu, K. Maslov et al., "Photoacoustic microscopy of blood pulse wave," Journal of biomedical optics, 17(7), 0705041-0705043 (2012).

[10] C. Yeh, B. Soetikno, S. Hu et al., "Three-dimensional arbitrary trajectory scanning photoacoustic microscopy," Journal of biophotonics, 9999, (2014).

[11] L. Li, C. Yeh, S. Hu et al., "Fully motorized optical-resolution photoacoustic microscopy," Optics Letters, 39(7), 2117-2120 (2014). 
[12] A. H. Shoeb, and J. V. Guttag, "Application of machine learning to epileptic seizure detection." 975-982.

[13] F. Pereira, T. Mitchell, and M. Botvinick, "Machine learning classifiers and fMRI: a tutorial overview," Neuroimage, 45(1), S199-S209 (2009).

[14] C. Yeh, B. Soetikno, S. Hu et al., "Microvascular quantification based on contourscanning photoacoustic microscopy," Journal of biomedical optics, 19(9), 096011096011 (2014).

[15] R. Zhang, L. Wang, J. Yao et al., "In vivo optically encoded photoacoustic flowgraphy," Optics Letters, 39(13), 3814-3817 (2014).

[16] E. L. Cussler, [Diffusion: mass transfer in fluid systems] Cambridge university press, (2009).

[17] D. E. Laughlin, K. P. Rodbell, O. Thomas et al., [Polycrystalline Metal and Magnetic Thin Films], (1999).

[18] R. N. Pittman, "Regulation of tissue oxygenation." 3, 1-100.

[19] P. F. Green, [Kinetics, transport, and structure in hard and soft materials] CRC Press, (2005).

[20] L. R. Adams, and I. Fatt, "The diffusion coefficient of human hemoglobin at high concentrations," Respiration Physiology, 2(3), 293-301 (1967).

[21] J. Yao, K. I. Maslov, Y. Zhang et al., "Label-free oxygen-metabolic photoacoustic microscopy in vivo," Journal of Biomedical Optics, 16(7), 076003-076003-11 (2011).

[22] Y. Anzai, [Pattern recognition and machine learning] Elsevier, (2012).

[23] T. Imai, B. Muz, C.-H. Yeh et al., "Direct measurement of hypoxia in a xenograft multiple myeloma model by optical-resolution photoacoustic microscopy," Cancer Biology \& Therapy, 1-5 (2017). 